\title{
Stochastic Geometry Analysis of Downlink Spectral and Energy Efficiency in Ultradense Heterogeneous Cellular Networks
}

\author{
Jiaqi Lei, Hongbin Chen (D), and Feng Zhao \\ Key Laboratory of Cognitive Radio and Information Processing, Guilin University of Electronic Technology, Guilin 541004, China \\ Correspondence should be addressed to Hongbin Chen; chbscut@guet.edu.cn
}

Received 18 October 2017; Revised 9 January 2018; Accepted 19 February 2018; Published 1 April 2018

Academic Editor: Jinglan Zhang

Copyright ( $\odot 2018$ Jiaqi Lei et al. This is an open access article distributed under the Creative Commons Attribution License, which permits unrestricted use, distribution, and reproduction in any medium, provided the original work is properly cited.

\begin{abstract}
The energy efficiency (EE) is a key metric of ultradense heterogeneous cellular networks (HCNs). Earlier works on the EE analysis of ultradense HCNs by using the stochastic geometry tool only focused on the impact of the base station density ratio and ignored the function of different tiers. In this paper, a two-tier ultradense HCN with small-cell base stations (SBSs) and user equipments (UEs) densely deployed in a traditional macrocell network is considered. Firstly, the performance of the ultradense HCN in terms of the association probability, average link spectral efficiency (SE), average downlink throughput, and average EE is theoretically analyzed by using the stochastic geometry tool. Then, the problem of maximizing the average EE while meeting minimum requirements of the average link SE and average downlink throughput experienced by UEs in macrocell and small-cell tiers is formulated. As it is difficult to obtain the explicit expression of average EE, impacts of the SBS density ratio and signal-tointerference-plus-noise ratio (SINR) threshold on the network performance are investigated through numerical simulations. Simulation results validate the accuracy of theoretical results and demonstrate that the maximum value of average EE can be achieved by optimizing the SBS density ratio and the SINR threshold.
\end{abstract}

\section{Introduction}

With the rapid growth of smart terminals, the wireless data traffic presented an exponential growth in recent years, which demands a much higher link throughput. However, latest wireless communication technologies have brought the point-to-point link throughput close to its theoretical limit, and the traditional homogeneous macrocell network cannot meet the enormous request on mobile services via the cell splitting technique anymore. In order to overcome these issues and provide a significant network performance leap, HCNs with the coexistence of macrocells and small cells have been introduced in the LTE-advanced standardization [1-3]. But in early HCNs, small cells were recommended as a complement for macrocells and were deployed in specific areas, such as hotspot and indoor scenarios. With the advancement of massive multiple-input multiple-output [4] and millimeter wave communication technologies, small cells were suggested to be densely deployed in all cellular network deployment scenarios [5]. So, the ultradense HCN, which has a much higher SBS density than ever, was viewed as one of the most promising paradigms to meet the $1000 \mathrm{x}$ wireless traffic volume increment in the next decade [6-11]. However, along with the increase of SBS density, the energy consumption of ultradense HCNs will inevitably increase [9-11]. Hence, the EE will be a major concern for designing environment-friendly ultradense HCNs. In this paper, endeavors are made to develop an analytical framework for evaluating the average link SE, average downlink throughput, and average EE in a two-tier ultradense $\mathrm{HCN}$, as well as providing guidelines for practical deployments.

1.1. Related Works and Motivation. In HCNs, SBSs are usually randomly deployed, which pose significant challenges to the theoretical analysis and simulation validation. Recent research works have shown that such randomly deployed cellular networks can be successfully analyzed by utilizing the stochastic geometry tool [12]. For instance, modeling HCNs by the spatial Poisson point process (PPP) can provide an effective and tractable approach to analyze the performance of $\mathrm{HCNs}$ in terms of the coverage 
probability [13], outage probability [14], and average ergodic rate [15]. Furthermore, Singh et al. [16] and Dhillon and Andrews [17] characterized the downlink rate distribution with the mean load approximation. Besides, there exist many other point processes that can capture the spatial distribution characteristics of cellular networks, such as the binomial point process [18], hard-core point process [19], Poisson cluster process [20], and so on. Nevertheless, the PPP model has the advantage of being more analytically tractable. Furthermore, abovementioned works modeled the spatial distribution of macrocell base stations (MBSs) by a random point process as well and ignored the function of different tiers or the relationship between them, which is impractical in actual situations. So, in this work, the spatial distribution of MBSs is modeled by a regular hexagonal structure, while the spatial distribution of SBSs is modeled by a PPP. The stochastic geometry tool and statistical average method are adopted to analyze the performance of the macrocell tier and small-cell tier, respectively.

On the other hand, universal frequency reuse is a key technology to meet the ever-increasing throughput demands, which is one of the main characteristics of HCNs [21-24]. That is, the available spectrum will be aggressively reused by all of the coexisting network tiers. However, in ultradense HCNs, the cross-tier interference between a macrocell and a small cell and the co-tier interference among small cells have become a severe problem. As a result, there has been a significant amount of research efforts on managing both cross-tier interference and co-tier interference in a two-tier HCN, which consists of a macrocell network overlaid with small cells $[25,26]$. In [25], the authors proposed a spectrum partitioning approach to avoid the cross-tier interference between the macrocell tier and the small-cell tier by using the orthogonal spectrum allocation. However, under a sparse small-cell deployment setting, this approach is clearly inefficient, and a much higher area SE can be attained if the spectrum sharing is allowed [26]. Inspired by earlier works mentioned above, the spectrum partitioning approach is adopted in the two-tier ultradense HCN with ultradense SBS deployment, which can obtain a higher network SE while significantly simplifying the theoretical analysis.

With respect to the EE, on one hand, significant efforts have been made to investigate the impact of base station density on the EE of HCNs. For example, the impact of base station deployment strategies especially the impact of base station density on the $\mathrm{EE}$ of ultradense $\mathrm{HCNs}$ was investigated in [27]. In [28], the authors analyzed the minimum base station density with the service outage constraint to minimize the network energy cost in $\mathrm{HCNs}$. On the other hand, some earlier works concentrated on the EE by using energy-saving technologies, such as traffic awareness $[29,30]$, collaborative transmission [31], and base station sleep scheduling [32]. In this work, we focus on the optimization of network parameters (SBS density ratio and SINR threshold) to maximize the average EE without considering these related technologies.

As far as we know, most earlier works that adopted random network models to analyze the performance of
HCNs focused on the network EE with respect to the SBS density ratio. In this work, the optimization problem of maximizing the average EE while meeting the average link SE and average downlink throughput requirements in the two-tier ultradense HCN is considered, and impacts of the SBS density ratio and SINR threshold on the network performance are investigated.

1.2. Contributions and Paper Organization. In this work, we provide a comprehensive average EE analysis of a two-tier ultradense HCN. Compared to earlier works, main contributions of this paper are summarized as follows:

(1) Taking different functions of macrocell and smallcell tiers into account, in a two-tier ultradense $\mathrm{HCN}$, a modified nearest association scheme is proposed, namely, a UE first decides whether to associate with its nearest SBS according to the received SINR. If its received SINR surpasses a threshold, the UE will associate with its nearest SBS. Otherwise, it will associate with its nearest MBS. Note that a UE associates with its nearest MBS without the limit of SINR threshold, since the macrocell tier is not interference-limited and the power of interference is controlled at a certain level which is comparable to the noise power.

(2) The association probability is used to measure the burden of macrocell and small-cell tiers, and the average link SE is used to measure the quality of communications. Besides, the average link SE and average downlink throughput are derived by using the statistical average method.

(3) To evaluate the average downlink throughput experienced by UEs in the small-cell tier, instead of using the average ergodic rate, the SINR threshold is taken into account, and impacts of the SBS density ratio and SINR threshold on the average EE are investigated. The aim is to provide a tractable approach to seek the optimum SBS density ratio and SINR threshold to maximize the average EE while meeting minimum requirements of the average link SE and average downlink throughput experienced by UEs in macrocell and small-cell tiers, which is meaningful to guide the actual deployment of ultradense HCNs.

The rest of this paper is organized as follows: in Section 2, the two-tier ultradense HCN model, channel model, power consumption model, and association scheme are described. In Section 3, the average link SE, average downlink throughput, and average EE are analytically derived, and the optimization problem is formulated. In Section 4, numerical simulation results are presented to validate theoretical ones. Finally, some concluding remarks are given in Section 5.

Notations: E[.] represents the expectation operator, $I_{x}$ represents the cumulative interference from all the other SBSs, $\mathrm{L}_{I_{x}}(\cdot)$ represents the Laplace transform of a random variable $I_{x}, P_{\mathrm{m}}$ and $P_{\mathrm{s}}$ denote the transmit powers of the MBS and SBS, respectively, $N_{\mathrm{m}}$ represents the number of 


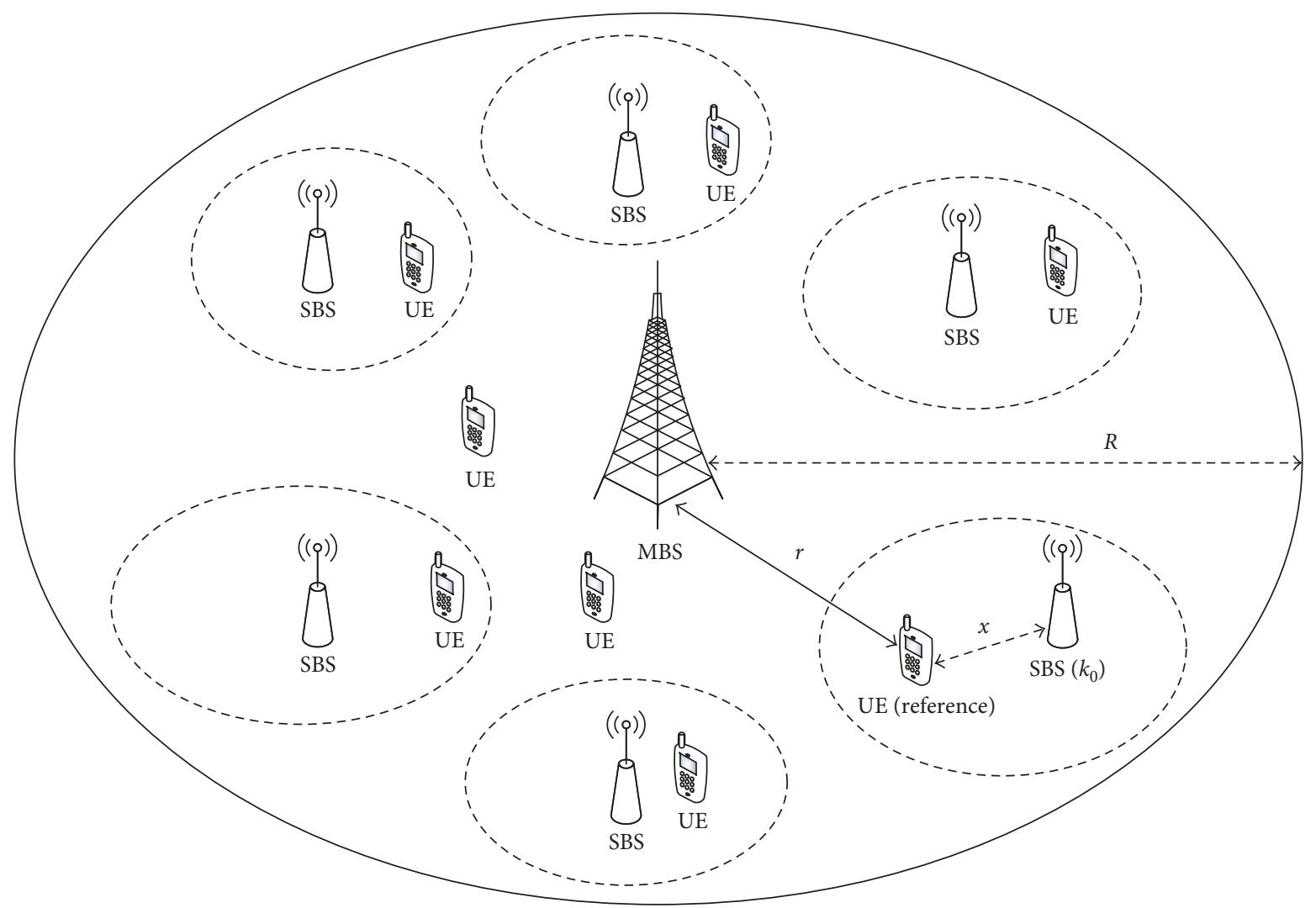

FIgURE 1: Illustration of a macrocell in the two-tier ultradense HCN.

UEs served by an MBS in the macrocell tier, $N_{\mathrm{s}}$ represents the number of UEs served by an SBS in the small-cell tier, the subscript $x$ represents the distance between the reference UE and its nearest SBS (denoted by $k_{0}$ ), and subscripts $m$ and $s$ represent the MBS and SBS, respectively.

\section{System Models}

2.1. Ultradense HCN Model. The ultradense HCN consists of a traditional homogeneous macrocell network and an ultradense small-cell network, in which SBSs constitute the small-cell tier and their spatial distribution is modeled as a homogeneous PPP $\Phi_{s}$ with the intensity $\lambda_{s}$, while MBSs constitute the macrocell tier and their spatial distribution follows a regular hexagonal structure since they are aimed to provide seamless coverage for a large area. Note that, in earlier works on performance analysis of cellular networks, the hexagonal structure was commonly substituted by a circular one for tractability. In addition, UEs follow another independent homogeneous PPP $\Phi_{\mathrm{u}}$ with the intensity $\lambda_{\mathrm{u}}$. In order to avoid the cross-tier interference, the spectrum partitioning approach is adopted in the ultradense HCN. Moreover, because the analyzing process is the same for different macrocells and we try to obtain the average throughput in a tractable way, we pick out one macrocell with the radius $R$ from the two-tier ultradense HCN to conduct the network performance analysis, as shown in Figure 1. In this macrocell, the MBS is located at the center, and the bandwidths $W_{1}$ and $W_{2}$ are dedicated for the MBS and SBS, respectively. Without loss of generality, it is assumed that a reference UE is located within the range of the MBS with a radial distance $r$, and all base stations and UEs are assumed to be equipped with a single antenna.

Since the impact of SBS density on the average EE for a given UE density will be investigated, the SBS density is normalized to $\lambda_{\mathrm{s}}=\rho \lambda_{\mathrm{u}}$, where $\rho$ is defined as the SBS density ratio. In this work, considering the feature of the ultradense $\mathrm{HCN}$ and referring to earlier works, let the value of $\rho$ vary within $0.01 \sim 0.3$. Note that $\rho$ can take other values in different scenarios.

2.2. Channel Model. The path loss and fading are taken into account when modeling the wireless channel. The path loss exponent is denoted as $\alpha>2$. The fading experienced by UEs is assumed to be Rayleigh fading with mean 1, and its power gain is denoted by $h$, which follows the exponential distribution, that is, $h \sim \exp (1)$.

In the macrocell tier, considering relevant techniques have been used to avoid the interference coming from adjacent MBSs, and in order to make the theoretical derivation tractable, it is assumed that the interference power coming from the other MBSs is comparable to the noise power $\sigma^{2}$, and the co-tier interference power $\xi^{2}=\omega \sigma^{2}$ is the same all over the macrocell tier [33], where the parameter $\omega$ reflects the level of interference power in the macrocell tier. In the small-cell tier, the universal frequency reuse scheme is adopted to improve the network throughput. As such, except 
the serving SBS of the reference UE, all the other SBSs are potential interferers.

Therefore, the SINR of the reference UE at a distance $r$ from its associated MBS can be expressed as

$$
\gamma_{1}(r)=\frac{P_{\mathrm{m}} h r^{-\alpha}}{\xi^{2}+\sigma^{2}},
$$

and the SINR of the reference UE at a distance $x$ from its associated SBS can be expressed as

$$
\gamma_{2}(x)=\frac{P_{s} h x^{-\alpha}}{I_{x}+\sigma^{2}}
$$

where $I_{x}=\sum_{j \in \Phi_{s}, j \neq k_{0}} P_{s} h_{j} v_{j}^{-\alpha}$ denotes the cumulative interference from all the other SBSs, in which the $j$ th SBS is at distance $v_{j}$ from the reference UE.

2.3. Power Consumption Model. Generally speaking, the power consumption in a base station includes two parts: static power consumption and transmit power consumption. The static power consumption in a base station is independent of the transmit power, while the transmit power consumption scales with the traffic load. In this work, the situation where all the base stations are fully loaded is supposed, so it is reasonable to assume that the transmit power consumption in a base station is independent of the traffic load but scales with the average radiated power.

Therefore, in order to compute the average power consumption of the macrocell in the two-tier ultradense $\mathrm{HCN}$, referring to [34], the power consumption in the MBS and the SBS can be simply written as linear expressions:

$$
\begin{gathered}
P_{\mathrm{m}, \text { tot }}=\Delta_{\mathrm{m}} P_{\mathrm{m}}+b_{\mathrm{m}}, \\
P_{\mathrm{s}, \mathrm{tot}}=\Delta_{\mathrm{s}} P_{\mathrm{s}}+b_{\mathrm{s}},
\end{gathered}
$$

where the coefficients $\Delta_{\mathrm{m}}$ and $\Delta_{\mathrm{s}}$, respectively, account for the power consumption that scales with the average radiated power due to amplifier and feeder losses. Static powers $b_{\mathrm{m}}$ and $b_{\mathrm{s}}$, respectively, include ones used for signal processing, battery backup, and cooling. So, the average power consumption over the area covered by the macrocell in the twotier ultradense HCN can be calculated as

$$
\bar{P}=\frac{P_{\mathrm{m}, \mathrm{tot}}+\lambda_{\mathrm{s}} \pi R^{2} P_{\mathrm{s}, \mathrm{tot}}}{\pi R^{2}} .
$$

2.4. Association Scheme. In earlier works [13, 27], the spatial distribution of base stations in each tier of the HCN was modeled as an independent homogeneous PPP, and an SINR threshold was set to control the UE access to different tiers. Besides, both of them modeled the multitier network with a simple combination of single-tier networks, and the function of different tiers seems to be the same, which is impractical in an actual HCN. Furthermore, there are always some UEs dropped from the $\mathrm{HCN}$, which means that the HCN cannot provide a seamless coverage.
In order to deal with problems mentioned above, in this work, a modified nearest association scheme is proposed, that is, a UE is covered by the small-cell tier when the SINR received from its nearest SBS is greater than the threshold $\beta$. Otherwise, it will be dropped from the small-cell tier and associate with the MBS.

Since UEs dropped from the small-cell tier will associate with the MBS, it is reasonable to use the association probability to characterize the burden of different tiers. The association probability is defined as $p=\mathrm{E}_{x}\left[P\left\{\gamma_{2}(x)>\beta \mid x\right\}\right]$, in which $P\left\{\gamma_{2}(x)>\beta \mid x\right\}$ is the probability that a UE at distance $x$ from its nearest SBS can achieve the target SINR $\beta$. It can be interpreted as (1) the average probability that a UE can achieve the target SINR $\beta$ when associating with its nearest SBS and (2) the average fraction of UEs who at any time can achieve the target SINR $\beta$ in the small-cell tier. According to [35], the association probability can be expressed as

$$
\begin{aligned}
p & =\mathrm{E}_{x}\left[P\left\{\gamma_{2}(x)>\beta \mid x\right\}\right] \\
& =\int_{x>0} P\left\{\gamma_{2}(x)>\beta \mid x\right\} f(x) d x \\
& =2 \pi \lambda_{\mathrm{s}} \int_{0}^{\infty} x \mathrm{~L}_{I_{x}}\left(\frac{\beta}{P_{\mathrm{s}} x^{-\alpha}}\right) e^{-\left(\beta \sigma^{2} / P_{\mathrm{s}} x^{-\alpha}\right)} e^{-\lambda_{\mathrm{s}} \pi x^{2}} d x,
\end{aligned}
$$

where $\mathrm{L}_{I_{x}}(s)=\mathrm{E}\left[e^{-s I_{x}}\right]=\exp \left(-2 \pi \lambda_{\mathrm{s}} \int_{x}^{\infty}\left(1-\left(1 /\left(s P_{\mathrm{s}} v^{-\alpha}+\right.\right.\right.\right.$ 1))) $v d v$ ) is the Laplace transform of the cumulative interference $I_{x}$ and $f(x)=2 \pi \lambda_{s} x e^{-\lambda_{s} \pi x^{2}}$ is the probability density function of $x$.

\section{Network Performance Analysis}

In this section, taking the association probability into account, the average link SE, average downlink throughput experienced by UEs in macrocell and small-cell tiers, and average EE are derived. Then, an optimization problem is formulated to maximize the average EE while meeting the average link SE and average downlink throughput requirements.

According to (1), the SINR of a UE associating with the MBS is relevant to the distance $r$. So, the average link SE experienced by UEs in the macrocell tier can be expressed as

$$
\begin{aligned}
\tau_{\mathrm{m}} & =\mathrm{E}\left[\log _{2}\left(1+\gamma_{1}(r)\right)\right] \\
& =\frac{1}{\pi R^{2}} \int_{0}^{2 \pi} \int_{0}^{R} \int_{0}^{\infty} \log _{2}\left(1+\frac{P_{\mathrm{m}} h r^{-\alpha}}{\sigma^{2}+\xi^{2}}\right) \mathrm{e}^{-h} r d h d r d \theta .
\end{aligned}
$$

From (2) and taking the SINR threshold $\beta$ into account, the average link SE experienced by UEs in the small-cell tier can be expressed as

$$
\begin{aligned}
\tau_{\mathrm{s}} & =\mathrm{E}\left[\log _{2}\left(1+\gamma_{2}(x)\right) \mid \gamma_{2}(x)>\beta\right] \\
& =\log _{2}(1+\beta)+\frac{1}{\ln (2)} \int_{0}^{\infty} \mathrm{A}(x) d x,
\end{aligned}
$$


where

$$
\mathrm{A}(x)=2 \pi \lambda_{\mathrm{s}} \int_{\beta}^{\infty} x \frac{\mathrm{L}_{I_{x}}\left(z / P_{\mathrm{s}} x^{-\alpha}\right) e^{-\left(z \sigma^{2} / P_{\mathrm{s}} x^{-\alpha}\right)}}{\mathrm{L}_{I_{x}}\left(\beta / P_{\mathrm{s}} x^{-\alpha}\right) e^{-\left(\beta \sigma^{2} / P_{\mathrm{s}} x^{-\alpha}\right)}} \frac{1}{z+1} e^{-\lambda_{\mathrm{s}} \pi x^{2}} d z
$$

Proof. Denote the random variable $\gamma_{2}(x)$ by $Y$. E $\left[\log _{2}(1+\right.$ $\left.\gamma_{2}(x)\right) \mid \gamma_{2}(x)>\beta$ ] can be calculated as follows [13]:

$$
\begin{aligned}
\mathrm{E}_{x}\left[\int_{0}^{\infty} \log _{2}(1+y) f_{Y}(y \mid Y>\beta) d y\right] \\
=\frac{1}{\ln (2)} \mathrm{E}_{x}\left[\int_{0}^{\infty} \int_{0}^{y} \frac{1}{(z+1)} f_{Y}(y \mid Y>\beta) d z d y\right] \\
=\frac{1}{\ln (2)} \mathrm{E}_{x}\left[\int_{0}^{\infty}\left(\int_{z}^{\infty} f_{Y}(y \mid Y>\beta) d y\right) \frac{1}{z+1} d z\right] \\
=\frac{1}{\ln (2)} \mathrm{E}_{x}\left[\int_{0}^{\infty} \frac{P(Y>z \mid Y>\beta)}{z+1} d z\right] \\
=\frac{1}{\ln (2)} \int_{0}^{\infty}\left(\int_{0}^{\infty} \frac{P(Y>z \mid Y>\beta)}{z+1} d z\right) f(x) d x \\
=\frac{1}{\ln (2)} \int_{0}^{\infty}\left(\int_{0}^{\infty} \frac{P(Y>z \mid Y>\beta)}{z+1} d z\right) 2 \pi \lambda_{\mathrm{s}} x e^{-\lambda_{s} \pi x^{2}} d x,
\end{aligned}
$$

where the first step follows the integral transformation and the second step follows from changing the order of integration, while the conditional complementary cumulative density function (CCDF) of $Y=\gamma_{2}(x)$ is written as follows:

$$
\begin{aligned}
& P(Y>z \mid Y>\beta) \\
& \quad=\frac{P(Y>z, Y>\beta)}{P(Y>\beta)} \\
& =\frac{P(Y>\max (z, \beta))}{P(Y>\beta)} \\
& \quad= \begin{cases}\frac{\mathrm{L}_{I_{x}}\left(z / P_{\mathrm{s}} x^{-\alpha}\right) e^{-\left(z \sigma^{2} / P_{\mathrm{s}} x^{-\alpha}\right)}}{\mathrm{L}_{I_{x}}\left(\beta / P_{\mathrm{s}} x^{-\alpha}\right) e^{-\left(\beta \sigma^{2} / P_{\mathrm{s}} x^{-\alpha}\right)}} & z>\beta \\
1, & \text { otherwise, }\end{cases}
\end{aligned}
$$

where $P(Y>\beta)=P\left\{\gamma_{2}(x)>\beta \mid x\right\}=\mathrm{L}_{I_{x}}\left(\beta / P_{s} x^{-\alpha}\right) e^{-\left(\beta \sigma^{2} / \mathrm{P}_{s} \mathrm{x}^{-\alpha}\right)}$.

It is clear that the average downlink throughput of a UE experienced in macrocell and small-cell tiers is mainly determined by the average number of UEs served by the MBS or its nearest SBS. According to the proposed association scheme, when the first interpretation of the association probability is adopted, that is, all the UEs have an equal probability to associate with the small-cell tier, referring to
[17], the spatial distribution of UEs that associate with the small-cell tier can be obtained by thinning the spatial distribution of all the UEs, which also follows a homogeneous PPP. In order to simplify the derivation, it is assumed that the spatial distribution of UEs served by the macrocell tier or the small-cell tier follows another independent homogeneous PPP, which means that the intensity of UEs served by the macrocell tier or the small-cell tier is identical in any area. Therefore, the average downlink throughput of UEs associating with different MBSs will be equal. Note that the independent thinning of the PPP of UEs that associate with the macrocell tier or the small-cell tier with a thinning probability is not considered. According to (5), in the macrocell with radius $R$, the average number of UEs served by the MBS is $\mathrm{E}\left[N_{\mathrm{m}}\right]=\pi R^{2}(1-p) \lambda_{\mathrm{u}}$. So, the average downlink throughput of a UE served by the MBS can be expressed as

$$
C_{\mathrm{m}}=\frac{W_{1} \tau_{\mathrm{m}}}{\mathrm{E}\left[N_{\mathrm{m}}\right]}
$$

With respect to UEs served by the small-cell tier, taking the association probability into account, the equivalent UE density served by the small-cell tier is $\lambda_{2}=p \lambda_{\mathrm{u}}$. The average downlink throughput of a UE served by its nearest SBS can be expressed as

$$
C_{s}=\frac{W_{2} \tau_{s}}{\mathrm{E}\left[N_{\mathrm{s}}\right]}
$$

where $\mathrm{E}\left[N_{\mathrm{s}}\right]=1+1.28\left(\lambda_{2} / \lambda_{\mathrm{s}}\right)$ is the average number of UEs served by an SBS [16]. As mentioned above, the average downlink throughput of a UE in the two-tier ultradense $\mathrm{HCN}$ can be written as

$$
\bar{C}=(1-p) C_{\mathrm{m}}+p C_{\mathrm{s}}
$$

The average EE is defined as the ratio of average area throughput to average area power consumption, and the average link SE is defined as the weighted sum of the average link SE obtained in macrocell and small-cell tiers. According to (4-7) and (13), the average EE and average link SE can be, respectively, expressed as

$$
\begin{aligned}
& \bar{\eta}_{\mathrm{EE}}=\frac{\lambda_{\mathrm{u}} \bar{C}}{\bar{P}}, \\
& \bar{\eta}_{\mathrm{SE}}=(1-p) \tau_{\mathrm{m}}+p \tau_{\mathrm{s}} .
\end{aligned}
$$

Finally, we try to maximize the average EE while meeting the minimum requirements of the average link SE and average downlink throughput. The optimization problem can be formulated as

$$
\begin{array}{ll}
\max & \bar{\eta}_{\mathrm{EE}} \\
\text { s.t. } & \bar{\eta}_{\mathrm{SE}} \geq \tau \\
& C_{\mathrm{s}} \geq C_{\mathrm{m}} \geq C_{\min },
\end{array}
$$

where $\tau$ is the minimum average link SE requirement and $C_{\text {min }}$ is the minimum requirement of the average downlink throughput experienced by UEs in macrocell and small-cell tiers. Note that (11), (12), (14), and (15) show that $C_{\mathrm{m}}, C_{\mathrm{s}}, \bar{\eta}_{\mathrm{EE}}$, 
TABLE 1: Simulation parameters.

\begin{tabular}{lcc}
\hline Parameter & Notation & Value \\
\hline Bandwidth & $W_{1}$ and $W_{2}$ & $10 \mathrm{MHz}$ and $10 \mathrm{MHz}$ \\
Path loss exponent & $\alpha$ & 3 \\
Noise power & $\sigma^{2}$ & $-100 \mathrm{dBm}$ \\
Coverage radius of the MBS & $R$ & $500 \mathrm{~m}$ \\
UE intensity & $\lambda_{\mathrm{u}}$ & $0.01 \mathrm{~m}^{-2}$ \\
Transmit power of the MBS & $P_{\mathrm{m}}$ & $40 \mathrm{~W}$ \\
Transmit power of the SBS & $P_{\mathrm{s}}$ & $2 \mathrm{~W}$ \\
Power parameters of the MBS & $\Delta_{\mathrm{m}}$ and $b_{\mathrm{m}}$ & $30 \mathrm{and} 500 \mathrm{~W}$ \\
Power parameters of the SBS & $\Delta_{\mathrm{s}}$ and $b_{\mathrm{s}}$ & $2 \mathrm{and} 10 \mathrm{~W}$ \\
Minimum average link SE requirement & $\tau$ & $3 \mathrm{bps} / \mathrm{Hz}$ \\
Minimum average downlink throughput requirement & $C_{\mathrm{min}}$ & $2 \times 10^{4} \mathrm{bps}$ \\
Level of interference power in the macrocell tier & $\omega$ & 10 \\
\hline
\end{tabular}

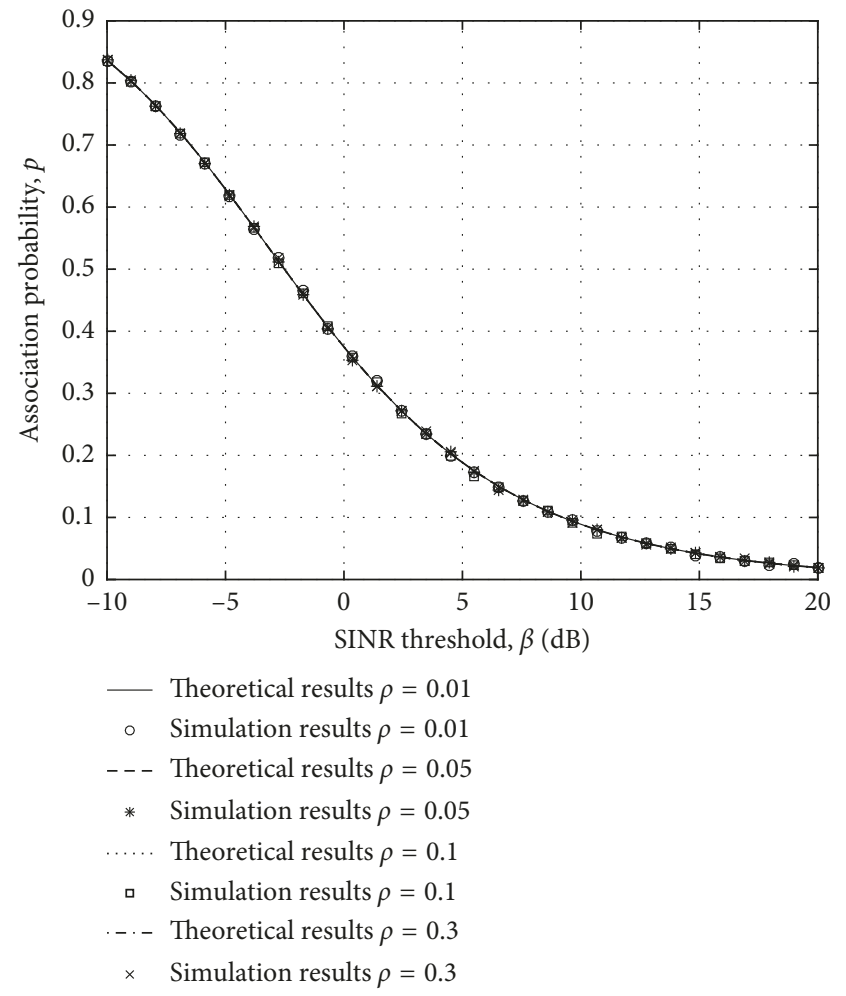

FIGURe 2: Association probability $p$ versus SINR threshold $\beta$.

and $\bar{\eta}_{\mathrm{SE}}$ are functions of the SBS density ratio $\rho$ and the SINR threshold $\beta$ on condition that values of $P_{\mathrm{m}}, P_{\mathrm{s}}, \alpha, R, \sigma^{2}, \omega, \lambda_{\mathrm{u}}$, $W_{1}$, and $W_{2}$ are all given. So, we can analyze impacts of $\rho$ and $\beta$ on $\bar{\eta}_{\mathrm{EE}}$ and try to seek optimum values of $\rho$ and $\beta$ to maximize $\bar{\eta}_{\mathrm{EE}}$ while meeting the requirements of $\bar{\eta}_{\mathrm{SE}}, C_{\mathrm{m}}$, and $C_{s}$. As it is difficult to obtain the explicit expression of $\bar{\eta}_{\mathrm{EE}}$, in the following section, extensive numerical simulations are conducted to evaluate impacts of $\rho$ and $\beta$ on $\bar{\eta}_{\mathrm{EE}}$.

\section{Simulation Results}

In the previous section, the average link SE, average downlink throughput, and average EE have been analytically derived. In this section, comparisons of Monte Carlo simulation results and theoretical results are presented to illustrate the

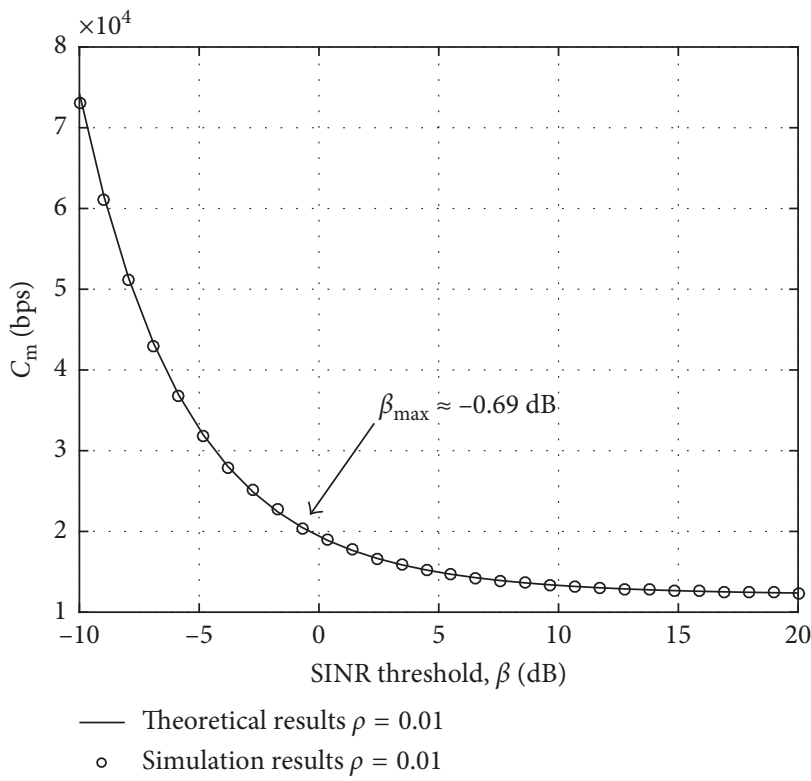

FIgURE 3: Average downlink throughput $C_{\mathrm{m}}$ of a UE served by the MBS versus SINR threshold $\beta$ when the SBS density ratio $\rho=0.01$.

performance of the two-tier ultradense $\mathrm{HCN}$, especially the average EE. Due to differences among system models as well as considered factors, results are not compared with those in earlier works. Simulation parameters are listed in Table 1.

As a key intermediate parameter in the two-tier ultradense $\mathrm{HCN}$, the association probability $p$ has an immediate impact on $C_{\mathrm{m}}, C_{\mathrm{s}}$, and $\bar{C}$, which is a function of $\rho$ and $\beta$ when values of $P_{s}, \alpha$, and $\sigma^{2}$ are given. So, impacts of $\rho$ and $\beta$ on $p$, $C_{\mathrm{m}}, C_{\mathrm{s}}$, and $\bar{C}$ are first investigated. Then, the values of $P_{\mathrm{m}}$, $P_{\mathrm{s}}, \alpha, R, \sigma^{2}, \omega, \lambda_{\mathrm{u}}, W_{1}$, and $W_{2}$ are all set, and impacts of $\rho$ and $\beta$ on $\bar{\eta}_{\mathrm{SE}}$ and $\bar{\eta}_{\mathrm{EE}}$ are investigated. Note that analytical results are numerically calculated with (5) and (11)-(15).

4.1. Impacts of $\rho$ and $\beta$ on $p, C_{\mathrm{m}}, C_{\mathrm{s}}$, and $\overline{\mathrm{C}}$. Figure 2 shows impacts of the SBS density ratio $\rho$ and SINR threshold $\beta$ on the association probability $p$. We can see that the SBS density ratio $\rho$ has little effect on the association probability $p$, while the association probability $p$ rapidly decreases with the increase of $\beta$. These results can be explained as follows: 


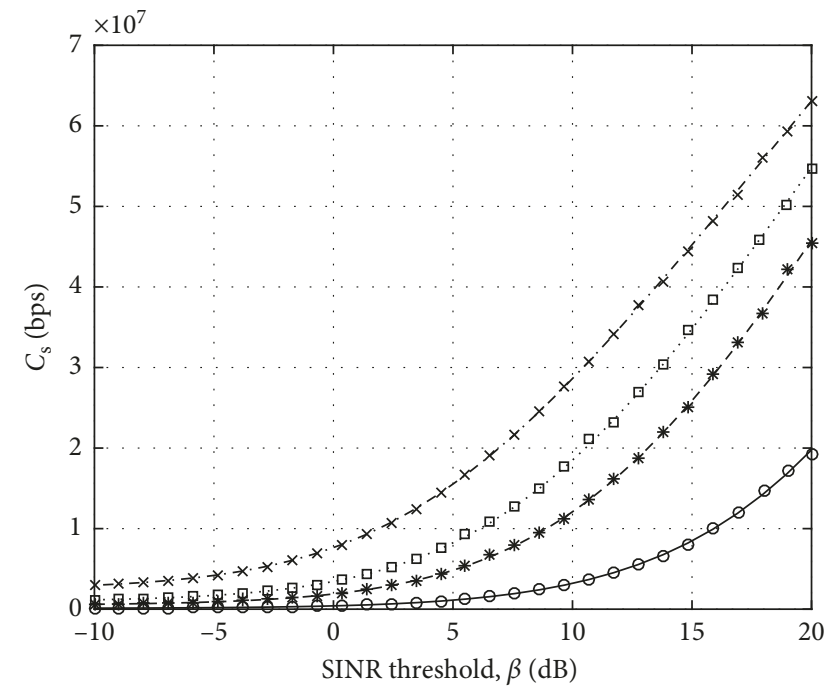

- Theoretical results $\rho=0.01$
$\quad$ Simulation results $\rho=0.01$
- - Theoretical results $\rho=0.05$
* Simulation results $\rho=0.05$
… Theoretical results $\rho=0.1$
- Simulation results $\rho=0.1$
_- Theoretical results $\rho=0.3$
× Simulation results $\rho=0.3$

FIgURE 4: Average downlink throughput $C_{\mathrm{s}}$ of a UE served by its nearest SBS versus SINR threshold $\beta$.

firstly, in the small-cell tier, the interference dominates the noise, which means that the noise power has little effect on the SINR [13]. Secondly, due to the random deployment of SBSs, the increase in signal power is exactly counterbalanced by the increase in interference power.

Since only the SINR threshold $\beta$ has an obvious effect on the association probability $p$, according to (11), $C_{\mathrm{m}}$ is determined by $\beta$ as well. So, we can observe the varying trend of the average downlink throughput of a UE served by the MBS, $C_{\mathrm{m}}$ versus $\beta$ for any given $\rho$. Figure 3 depicts the impact of the SINR threshold $\beta$ on $C_{\mathrm{m}}$ with $\rho=0.01$. From this figure, we can observe that $C_{\mathrm{m}}$ rapidly decreases with the increase of $\beta$. It can be interpreted that more UEs drop from the small-cell tier and associate with the MBS due to the increase of $\beta$. With simulation parameters listed in Table 1 , we can obtain the maximum value of $\beta$ as $\beta_{\max } \approx-0.69 \mathrm{~dB}$.

Figure 4 depicts the impact of the SINR threshold $\beta$ on the average downlink throughput $C_{\mathrm{s}}$ of a UE served by its nearest SBS for different values of $\rho$. From this figure, we can observe that $C_{\mathrm{s}}$ increases with $\beta$ and $\rho$. The reason is that a UE served by its nearest SBS shares its serving base station with a smaller number of the other UEs and experiences a higher SINR.

Figures 5 and 6 show impacts of the SBS density ratio $\rho$ and SINR threshold $\beta$ on the average downlink throughput $\bar{C}$, respectively. We can see that simulation results coincide with theoretical results, which validates the expression of the average downlink throughput in (13). From Figure 5, we can observe that the average downlink throughput increases with $\rho$ for any curve; it can be interpreted that less UEs are severed by its

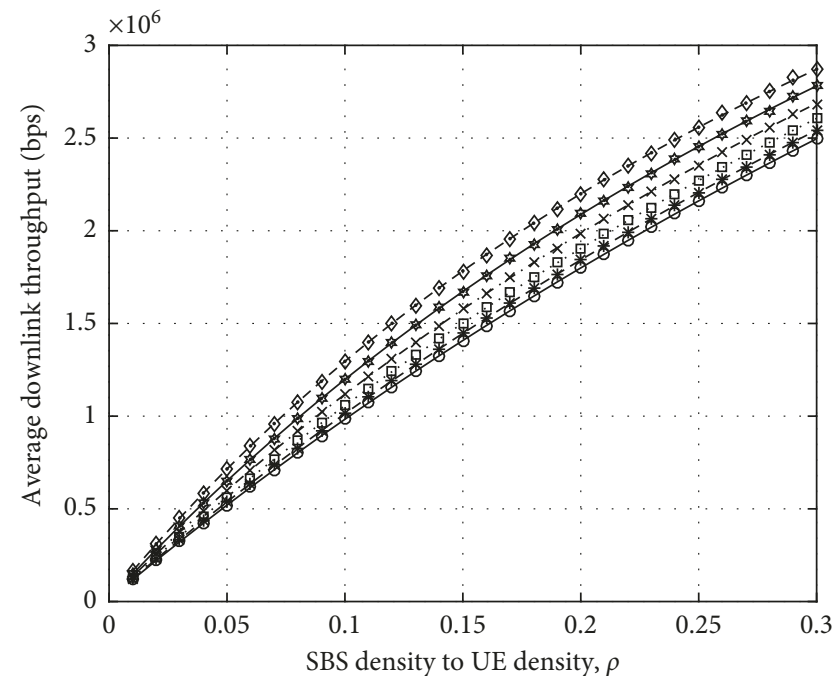

$$
\begin{aligned}
& \text { - Theoretical } \beta=-10 \mathrm{~dB} \quad . .- \text { Theoretical } \beta=-4 \mathrm{~dB} \\
& \text { - Simulation } \beta=-10 \mathrm{~dB} \quad \times \quad \text { Simulation } \beta=-4 \mathrm{~dB} \\
& -- \text { Theoretical } \beta=-8 \mathrm{~dB} \quad \longrightarrow \text { Theoretical } \beta=-2 \mathrm{~dB} \\
& \text { * Simulation } \beta=-8 \mathrm{~dB} \quad \forall \quad \text { Simulation } \beta=-2 \mathrm{~dB} \\
& \text {.... Theoretical } \beta=-6 \mathrm{~dB} \quad \ldots \text { Theoretical } \beta=0 \mathrm{~dB} \\
& \text { 口 Simulation } \beta=-6 \mathrm{~dB} \quad \diamond \quad \text { Simulation } \beta=0 \mathrm{~dB}
\end{aligned}
$$

FIgURE 5: Average downlink throughput $\bar{C}$ versus SBS density ratio $\rho$.

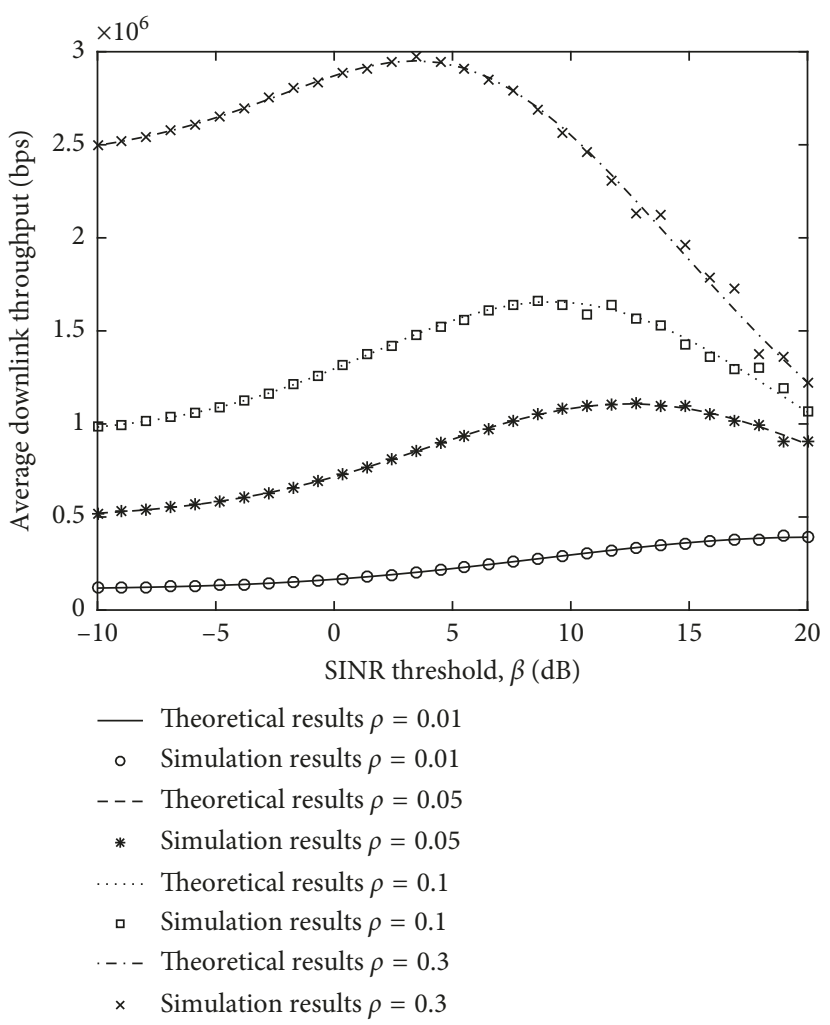

FIgURE 6: Average downlink throughput $\bar{C}$ versus SINR threshold $\beta$.

nearest SBS when $\rho$ increases, and it is sure to approach a certain limit when each small cell has only one active UE. From Figure 6, we can observe that if the SBS density ratio $\rho$ is 


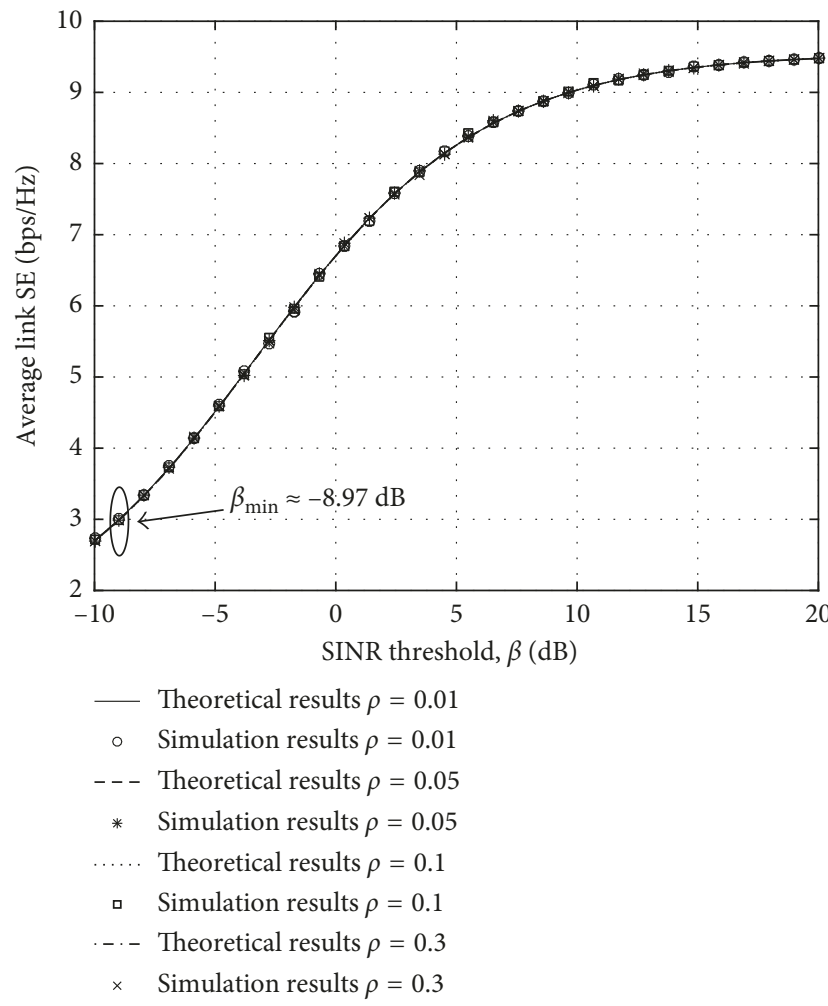

Figure 7: Average link SE $\bar{\eta}_{\text {SE }}$ versus SINR threshold $\beta$.

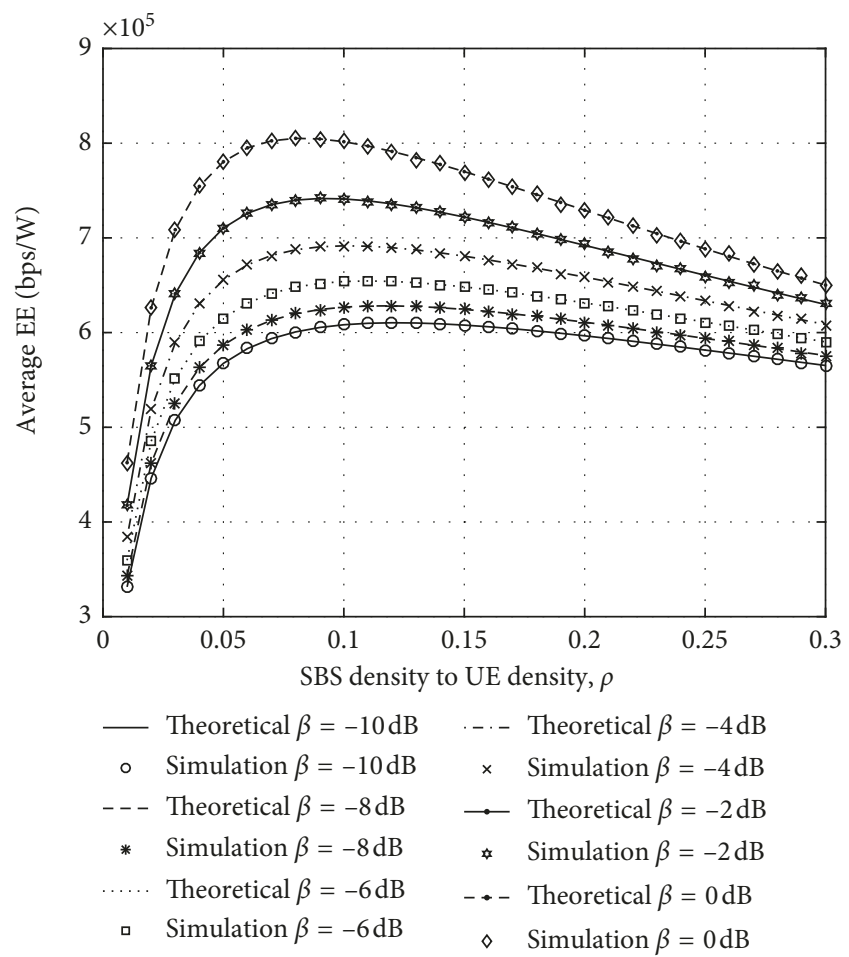

FIgURE 8: Average EE $\bar{\eta}_{\mathrm{EE}}$ versus SBS density ratio $\rho$.

given, we can seek an optimum $\beta$ to get the maximum average downlink throughput, which shows that $\beta$ plays the role to adjust the traffic burden between macrocell and small-cell tiers.

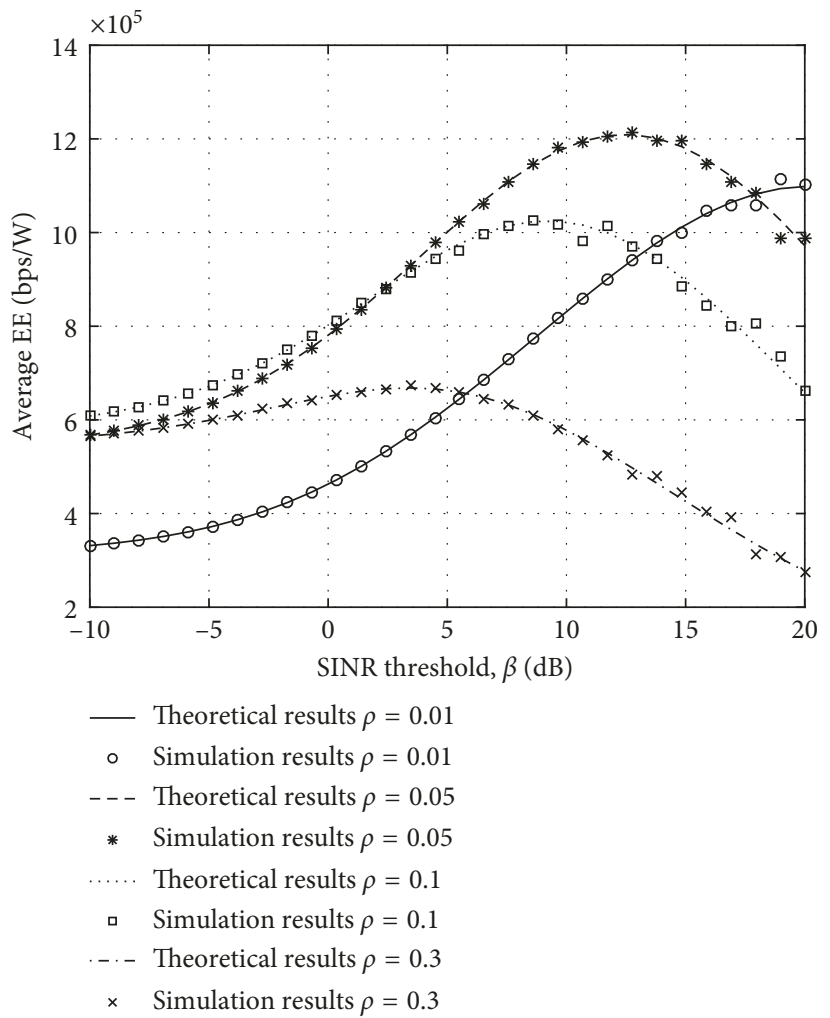

FIgURE 9: Average EE $\bar{\eta}_{\mathrm{EE}}$ versus SINR threshold $\beta$.

4.2. Impacts of $\rho$ and $\beta$ on $\bar{\eta}_{S E}$ and $\bar{\eta}_{E E}$. Impacts of the SBS density ratio $\rho$ and SINR threshold $\beta$ on the average link SE $\bar{\eta}_{\mathrm{SE}}$ and average $\mathrm{EE} \bar{\eta}_{\mathrm{EE}}$ are further simulated, which are shown in Figures 7-9. From Figure 7, we can see that the average link SE $\bar{\eta}_{\mathrm{SE}}$ first increases and finally saturates with the increase of SINR threshold $\beta$, while the SBS density ratio $\rho$ has little effect on $\bar{\eta}_{\mathrm{SE}}$. Combining with results shown in Figure 6 , we can conclude that a UE in the macrocell tier experiences a higher SINR, while in the microcell tier, a UE usually obtains a higher throughput. With the parameter $\tau$ listed in Table 1 , we can obtain the minimum value of $\beta$ as $\beta_{\text {min }} \approx-8.97 \mathrm{~dB}$. From Figure 8 , we can observe that the average $\mathrm{EE} \bar{\eta}_{\mathrm{EE}}$ first increases and then decreases with the increase of SBS density ratio $\rho$. It can be interpreted that the power consumption caused by the increment of SBSs surpasses the gain they bring to the throughput, which indicates that the SBS density ratio $\rho$ should be carefully designed. From Figure 9, we can observe that there exists an optimum $\beta$ to reach the maximum value of average $\mathrm{EE}$ for a given $\rho$. According to Figure 8 and considering the requirements of $\bar{\eta}_{\mathrm{SE}}, C_{\mathrm{m}}$, and $C_{\mathrm{s}}$, we can obtain optimum values of the SBS density ratio and SINR threshold as $\rho \approx 0.08$ and $\beta \approx-0.69 \mathrm{~dB}$, respectively, to make the two-tier ultradense $\mathrm{HCN}$ most energy-efficient.

From above simulation results, we can find that $\beta>-0.69 \mathrm{~dB}$ makes the average EE higher. However, it makes the throughput of UEs served by the MBS too low to bear. Besides, it is clear that further increasing $\rho$ or $\beta$ would not benefit the average EE. In order to further improve the average EE while meeting the minimum requirements of the average 
link SE and average downlink throughput in the two-tier ultradense $\mathrm{HCN}$, more techniques like interference management and base station cooperation need to be incorporated, which will be conducted in our future work.

\section{Conclusions}

In this paper, impacts of the SBS density ratio $\rho$ and SINR threshold $\beta$ on the performance of a two-tier ultradense HCN have been studied by using the stochastic geometry tool. In addition to theoretically deriving expressions of the average link SE, average downlink throughput, and average $\mathrm{EE}$, an optimization problem was formulated to maximize the average EE while meeting the average link SE and average downlink throughput requirements. Simulation results indicate that there exists an optimum value of $\rho$ or $\beta$ to maximize the average EE. But if one would like to achieve a high average EE while guaranteeing the performance of the two-tier ultradense $\mathrm{HCN}$, such as meeting minimum requirements of the average link SE and average downlink throughput, both $\rho$ and $\beta$ should be carefully designed. From simulation results, we can observe that, with parameter settings listed in Table 1 , the SBS density ratio as $\rho \approx 0.08$ and the SINR threshold as $\beta \approx-0.69 \mathrm{~dB}$ can make the two-tier ultradense HCN most energy-efficient. In brief, this work is capable of providing theoretical insights for the energyefficient planning of ultradense HCNs.

\section{Conflicts of Interest}

The authors declare that there are no conflicts of interest regarding the publication of this paper.

\section{Acknowledgments}

This work was supported by the National Natural Science Foundation of China (61671165 and 61471135), the Guangxi Natural Science Foundation (2015GXNSFBB139007 and 2016GXNSFGA380009), the Fund of Key Laboratory of Cognitive Radio and Information Processing (Guilin University of Electronic Technology), Ministry of Education, China, the Guangxi Key Laboratory of Wireless Wideband Communication and Signal Processing (CRKL160105 and CRKL170101), and the Innovation Project of GUET Graduate Education (2016YJCX91 and 2017YJCX27).

\section{References}

[1] A. Damnjanovic, J. Montojo, Y. Wei et al., "A survey on 3GPP heterogeneous networks," IEEE Wireless Communications, vol. 18, no. 3, pp. 10-21, 2011.

[2] D. Lopez-Perez, I. Guvenc, G. de la Roche, M. Kountouris, T. Q. S. Quek, and J. Zhang, "Enhanced intercell interference coordination challenges in heterogeneous networks," IEEE Wireless Communications, vol. 18, no. 3, pp. 22-30, 2011.

[3] A. Ghosh, N. Mangalvedhe, R. Ratasuk et al., "Heterogeneous cellular networks: from theory to practice," IEEE Communications Magazine, vol. 50, no. 6, pp. 54-64, 2012.

[4] W. Tan, M. Matthaiou, S. Jin, and X. Li, "Spectral efficiency of DFT-based processing hybrid architectures in massive
MIMO," IEEE Wireless Communications Letters, vol. 6, no. 5, pp. 586-589, 2017.

[5] X. Ge, S. Tu, G. Mao, C. Wang, and T. Han, "5G ultra-dense cellular networks," IEEE Wireless Communications, vol. 23, no. 1, pp. 72-79, 2016.

[6] N. Bhushan, J. Li, D. Malladi et al., "Network densification: the dominant theme for wireless evolution into 5G," IEEE Communications Magazine, vol. 52, no. 2, pp. 82-89, 2014.

[7] I. Hwang, B. Song, and S. S. Soliman, "A holistic view on hyper-dense heterogeneous and small cell networks," IEEE Communications Magazine, vol. 51, no. 6, pp. 20-27, 2013.

[8] Z. Wang, B. Hu, X. Wang, and S. Chen, "Interference pricing in 5G ultra-dense small cell networks: a Stackelberg game approach," IET Communications, vol. 10, no. 15, pp. 1865$1872,2016$.

[9] J. Liu and S. Sun, "Energy efficiency analysis of cache-enabled cooperative dense small cell networks," IET Communications, vol. 11, no. 4, pp. 477-482, 2017.

[10] S. F. Yunas, M. Valkama, and J. Niemela, "Spectral and energy efficiency of ultra-dense networks under different deployment strategies," IEEE Communications Magazine, vol. 53, no. 1, pp. 90-100, 2015.

[11] C. Yang, J. Li, Q. Ni, A. Anpalagan, and M. Guizani, "Interference-aware energy efficiency maximization in 5G ultra-dense networks," IEEE Transactions on Communications, vol. 65, no. 2, pp. 728-739, 2017.

[12] H. ElSawy, E. Hossain, and M. Haenggi, "Stochastic geometry for modeling, analysis, and design of multi-tier and cognitive cellular wireless networks: a survey," IEEE Communications Surveys \& Tutorials, vol. 15, no. 3, pp. 996-1019, 2013.

[13] H. S. Dhillon, R. K. Ganti, F. Baccelli, and J. G. Andrews, "Modeling and analysis of K-tier downlink heterogeneous cellular networks," IEEE Journal on Selected Areas in Communications, vol. 30, no. 3, pp. 550-560, 2012.

[14] H. Jo, Y. J. Sang, P. Xia, and J. G. Andrews, "Heterogeneous cellular networks with flexible cell association: a comprehensive downlink SINR analysis," IEEE Transactions on Wireless Communications, vol. 11, no. 10, pp. 3484-3495, 2012.

[15] M. Di Renzo, A. Guidotti, and G. E. Corazza, “Average rate of downlink heterogeneous cellular networks over generalized fading channels: a stochastic geometry approach," IEEE Transactions on Communications, vol. 61, no. 7, pp. 30503071, 2013.

[16] S. Singh, H. S. Dhillon, and J. G. Andrews, "Offloading in heterogeneous networks: modeling, analysis, and design insights," IEEE Transactions on Wireless Communications, vol. 12 , no. 5, pp. 2484-2497, 2013.

[17] H. S. Dhillon and J. G. Andrews, "Downlink rate distribution in heterogeneous cellular networks under generalized cell selection," IEEE Wireless Communications Letters, vol. 3, no. 1, pp. 42-45, 2014.

[18] M. Afshang and H. S. Dhillon, "Fundamentals of modeling finite wireless networks using binomial point process," IEEE Transactions on Wireless Communications, vol. 16, no. 5, pp. 3355-3370, 2017.

[19] Y. Li, F. Baccelli, J. G. Andrews, T. D. Novlan, and J. Zhang, "Modeling and analyzing the coexistence of Wi-Fi and LTE in unlicensed spectrum," IEEE Transactions on Wireless Communications, vol. 15, no. 9, pp. 6310-6326, 2016.

[20] C. Saha, M. Afshang, and H. S. Dhillon, "3GPP-inspired HetNet model using Poisson cluster process: sum-product functionals and downlink coverage," IEEE Transactions on Communications, no. 99, p. 1, 2017. 
[21] P. Lin, J. Zhang, Y. Chen, and Q. Zhang, "Macro-femto heterogeneous network deployment and management: from business models to technical solutions," IEEE Wireless Communications, vol. 18, no. 3, pp. 64-70, 2011.

[22] J. G. Andrews, H. Claussen, M. Dohler, S. Rangan, and M. C. Reed, "Femtocells: past, present, and future," IEEE Journal on Selected Areas in Communications, vol. 30, no. 3, pp. 497-508, 2012.

[23] S. Cheng, S. Lien, F. Chu, and K. Chen, "On exploiting cognitive radio to mitigate interference in macro/femto heterogeneous networks," IEEE Wireless Communications, vol. 18, no. 3, pp. 40-47, 2011.

[24] N. Saquib, E. Hossain, L. B. Le, and D. I. Kim, "Interference management in OFDMA femtocell networks: issues and approaches," IEEE Wireless Communications, vol. 19, no. 3, pp. 86-95, 2012.

[25] V. Chandrasekhar and J. G. Andrews, "Spectrum allocation in tiered cellular networks," IEEE Transactions on Communications, vol. 57, no. 10, pp. 3059-3068, 2009.

[26] W. C. Cheung, T. Q. S. Quek, and M. Kountouris, “Throughput optimization, spectrum allocation, and access control in twotier femtocell networks," IEEE Journal on Selected Areas in Communications, vol. 30, no. 3, pp. 561-574, 2012.

[27] T. Zhang, J. Zhao, L. An, and D. Liu, "Energy efficiency of base station deployment in ultra dense HetNets: a stochastic geometry analysis," IEEE Wireless Communications Letters, vol. 5, no. 2, pp. 184-187, 2016.

[28] D. Cao, S. Zhou, and Z. Niu, "Optimal combination of base station densities for energy-efficient two-tier heterogeneous cellular networks," IEEE Transactions on Wireless Communications, vol. 12, no. 9, pp. 4350-4362, 2013.

[29] Z. Li, D. Grace, and P. Mitchell, "Traffic-aware cell management for green ultradense small-cell networks," IEEE Transactions on Vehicular Technology, vol. 66, no. 3, pp. 2600-2614, 2017.

[30] L. Xiang, X. Ge, C. Wang, F. Y. Li, and F. Reichert, "Energy efficiency evaluation of cellular networks based on spatial distributions of traffic load and power consumption," IEEE Transactions on Wireless Communications, vol. 12, no. 3, pp. 961-973, 2013.

[31] C. Yang, J. Li, and M. Guizani, "Cooperation for spectral and energy efficiency in ultra-dense small cell networks," IEEE Wireless Communications, vol. 23, no. 1, pp. 64-71, 2016.

[32] Y. S. Soh, T. Q. S. Quek, M. Kountouris, and H. Shin, "Energy efficient heterogeneous cellular networks," IEEE Journal on Selected Areas in Communications, vol. 31, no. 5, pp. 840-850, 2013.

[33] H. Chen, W. Chen, F. Zhao, L. Fan, and H. Zhang, "Stochastic geometry analysis of downlink energy efficiency for a relay deployment scheme in relay-assisted cellular networks," Telecommunication Systems, vol. 63, no. 2, pp. 263-273, 2016.

[34] J. Peng, P. Hong, and K. Xue, "Energy-aware cellular deployment strategy under coverage performance constraints," IEEE Transactions on Wireless Communications, vol. 14, no. 1, pp. 69-80, 2015.

[35] J. G. Andrews, F. Baccelli, and R. K. Ganti, "A tractable approach to coverage and rate in cellular networks," IEEE Transactions on Communications, vol. 59, no. 11, pp. 3122-3134, 2011. 


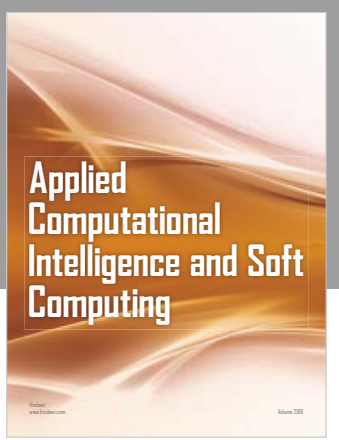

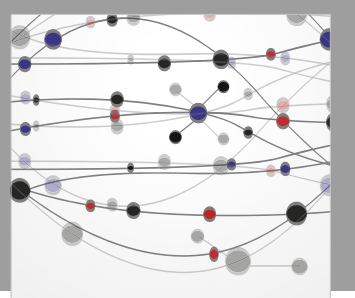

The Scientific World Journal
Submit your manuscripts at

Computing
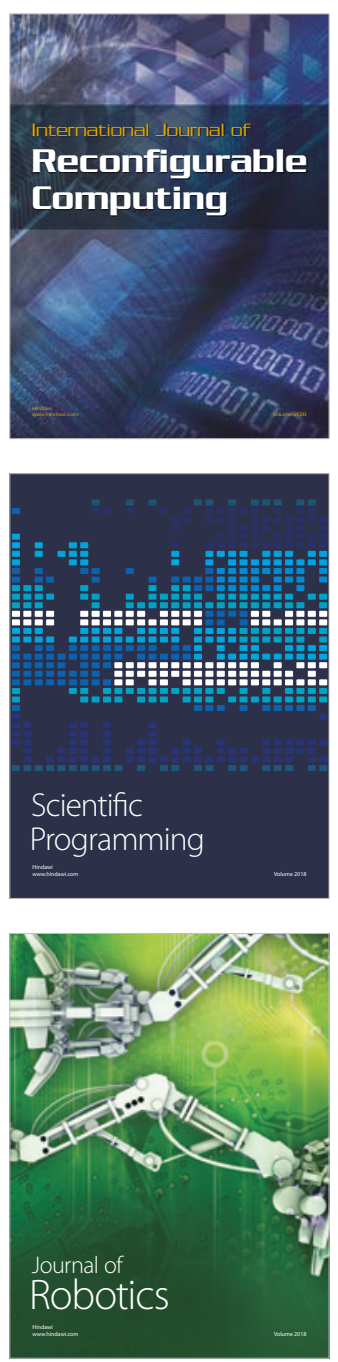

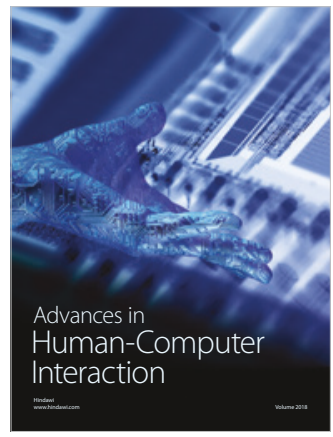

Human-Compute

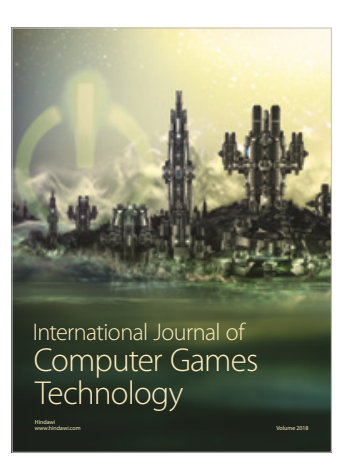

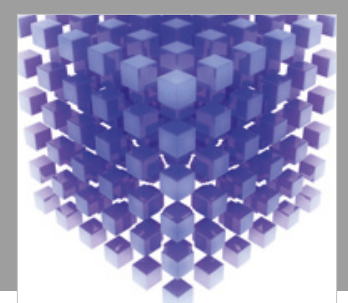

Mathematical Problems in Engineering

\section{Engincering}
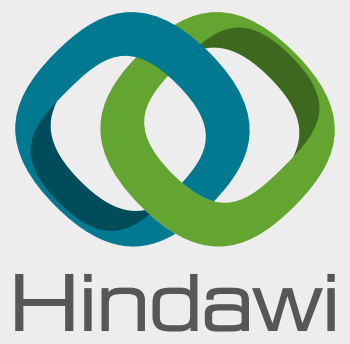

www.hindawi.com
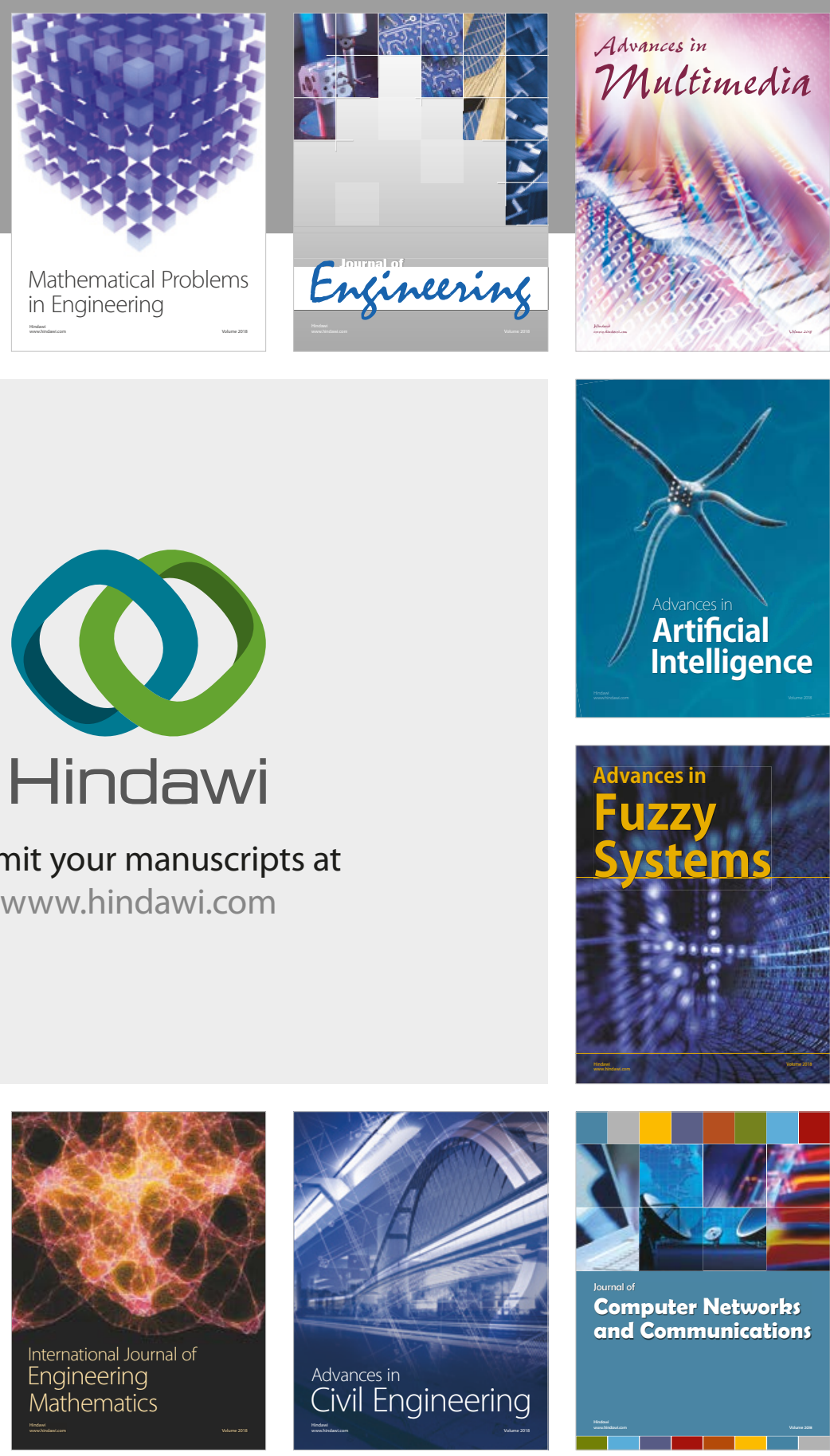

Computer Networks and Communications

Multimedia
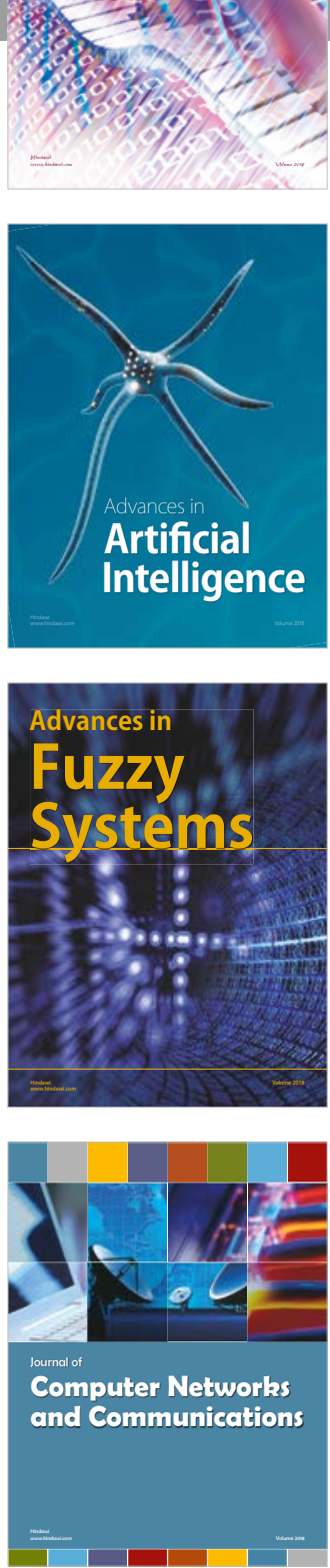

Advances in

Modelling \&

Simulation

in Engineering

interaction

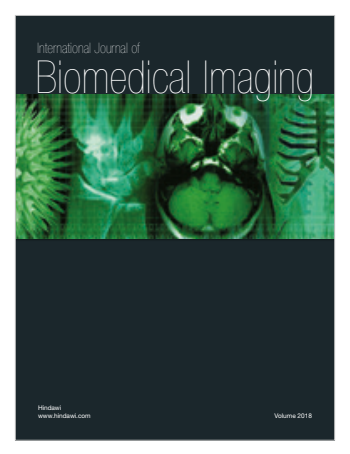

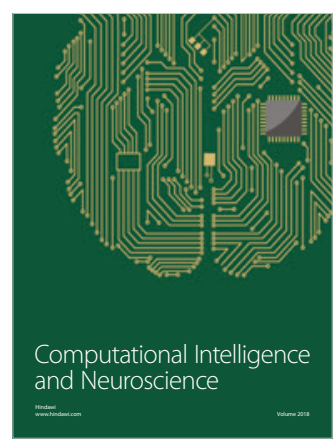

